\title{
Corrigendum: Diversity and Function of Wolf Spider Gut Microbiota Revealed by Shotgun Metagenomics
}

\author{
Runbiao $W^{1,2}$, Luyu Wang ${ }^{1}$, Jianping $X i e^{1}$ and Zhisheng Zhang ${ }^{1 *}$ \\ ${ }^{1}$ Key Laboratory of Eco-environments in Three Gorges Reservoir Region (Ministry of Education), School of Life Sciences, \\ Southwest University, Chongqing, China, ${ }^{2}$ Key Laboratory of Freshwater Fish Reproduction and Development (Ministry of \\ Education), School of Life Sciences, Southwest University, Chongqing, China
}

Keywords: shotgun metagenomic sequencing, spiders, host-bacterial interaction, symbiosis, microbiome

\section{A Corrigendum on}

Diversity and Function of Wolf Spider Gut Microbiota Revealed by Shotgun Metagenomics by Wu, R., Wang, L., Xie, J., and Zhang, Z. (2021). Front. Microbiol. 12:758794. doi: $10.3389 /$ fmicb.2021.758794

In the original article Abstract, there was the following error: Approximately 27.3\% of the gut microbiota of P. agraria comprises Proteobacteria, and approximately $34.5 \%$ of the gut microbiota of P. laura comprises Firmicutes.

The following correction has been applied:

Approximately $27.3 \%$ of the gut microbiota of $P$. agraria comprises Proteobacteria, and approximately $34.4 \%$ of the gut microbiota of $P$. laura comprises Firmicutes.

In the original article Figure 4 legend, contained the following errors: Ras guanyl-nuxleotide

Edited and reviewed by: Olivier Duron, Centre National de la Recherche Scientifique (CNRS), France

*Correspondence: Zhisheng Zhang zhangzs327@qq.com orcid.org/0000-0002-9304-1789

Specialty section: This article was submitted to Microbial Symbioses, a section of the journal Frontiers in Microbiology

Received: 05 January 2022 Accepted: 11 January 2022 Published: 02 February 2022

Citation: Wu R, Wang L, Xie J and Zhang Z (2022) Corrigendum: Diversity and

Function of Wolf Spider Gut Microbiota Revealed by Shotgun Metagenomics.

Front. Microbiol. 13:849170. doi: $10.3389 /$ fmicb.2022.849170 exchange factor activity should be changed to "Ras guanyl-nucleotide exchange factor activity," and intracellular receptor signaling patway should be changed to "intracellular receptor signaling pathway."

In the original article, MATERIALS AND METHODS, Assembly-Free Metagenomic Profiling, Metagenome Assembly, Gene Prediction, and Annotation: there was the following error: Quality control of metagenomic sequencing data was performed using MultiQC v1.5 (Ewels et al., 2016) with parameter $-t 20$.

The following correction has been applied: Quality control of metagenomic sequencing data was performed using FastQC v0.11.8 ${ }^{1}$ with parameter -t 20 and MultiQC v1.10.1 (Ewels et al., 2016) with parameter $-\mathrm{d}$.

${ }^{1}$ http://www.bioinformatics.babraham.ac.uk/projects/fastqc/

In the original article RESULTS, Identification of Microbiota From the Reads of Spider Metagenomes, there were the following errors: The most abundant gut microbiota in P. agraria were bacteria, accounting for $99.68 \%$, followed by archaea and viruses accounting for 0.3 and $0.02 \%$, respectively (Figure $2 A$ ). In the gut of P. laura, bacteria, archaea, and viruses accounted for $97.4,2$, and $0.6 \%$, respectively (Figure $2 B$ ).

In both species, bacteria from the phylum Proteobacteria were most abundant, accounting for approximately $27.3 \%(127,304 / 466,552)$ in P. agraria and $34.5 \%(186,388 / 542,484)$ in $P$. laura (Figure 2C).

The following correction has been applied:

The most abundant gut microbiota in P. agraria were bacteria, accounting for $99.64 \%$, followed by archaea and viruses accounting for 0.34 and $0.02 \%$, respectively (Figure 2A). In the gut of $P$. laura, bacteria, archaea, and viruses accounted for 97.56, 1.8, and 0.64\%, respectively (Figure 2B). 
In both species, bacteria from the phylum Proteobacteria were most abundant, accounting for approximately $27.3 \%$ $(127,304 / 466,552)$ in P. agraria and $34.4 \%(186,388 / 542,484)$ in P. laura (Figure 2C).

The authors apologize for the errors and state that this does not change the scientific conclusions of the article in any way. The original article has been updated.

Publisher's Note: All claims expressed in this article are solely those of the authors and do not necessarily represent those of their affiliated organizations, or those of the publisher, the editors and the reviewers Any product that may be evaluated in this article, or claim that may be made by its manufacturer, is not guaranteed or endorsed by the publisher.

Copyright (c) $2022 \mathrm{Wu}$, Wang, Xie and Zhang. This is an open-access article distributed under the terms of the Creative Commons Attribution License (CC BY). The use, distribution or reproduction in other forums is permitted, provided the original author(s) and the copyright owner(s) are credited and that the original publication in this journal is cited, in accordance with accepted academic practice. No use, distribution or reproduction is permitted which does not comply with these terms. 


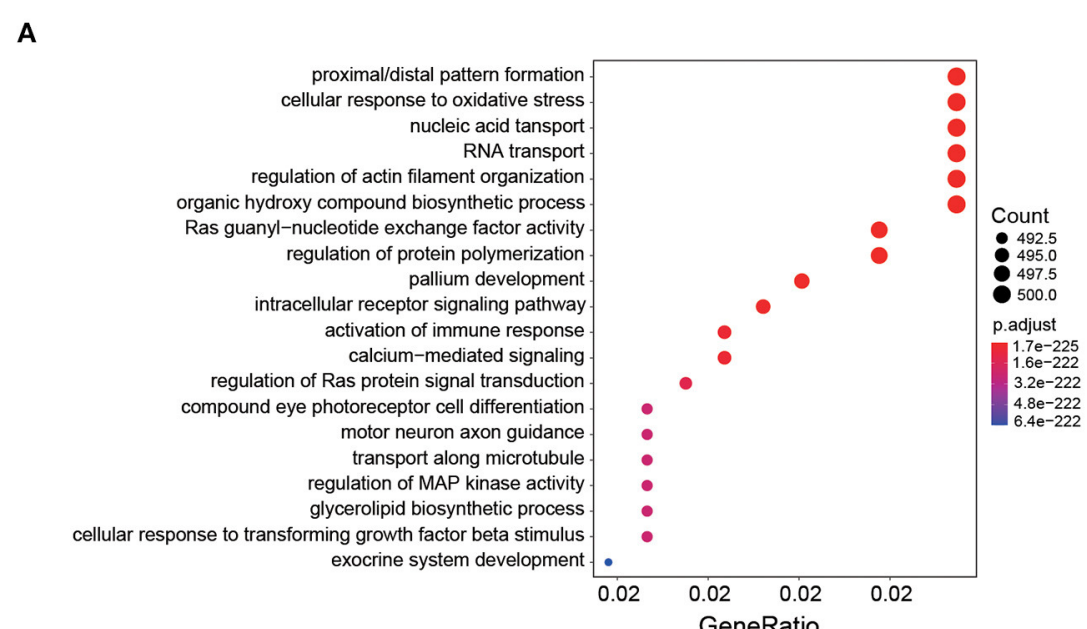

C
B

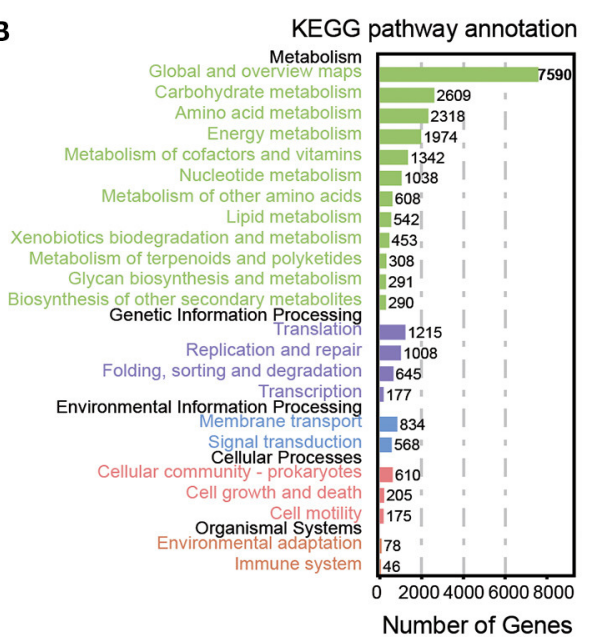

\section{$\square P$. agraria $\square P$. laura}
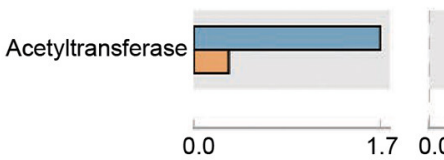

Mean proportion (\%)
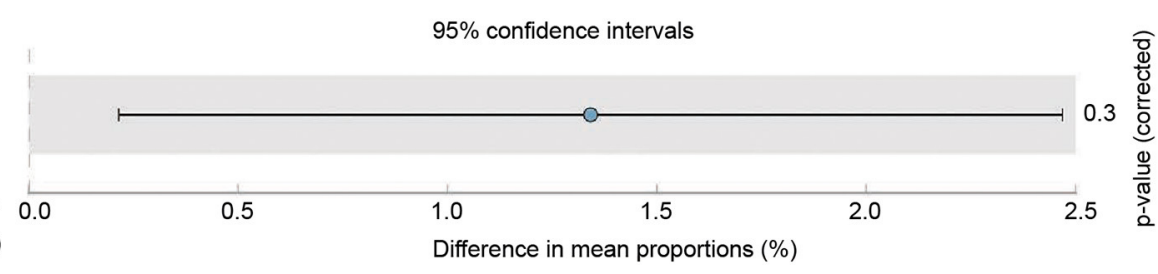

D

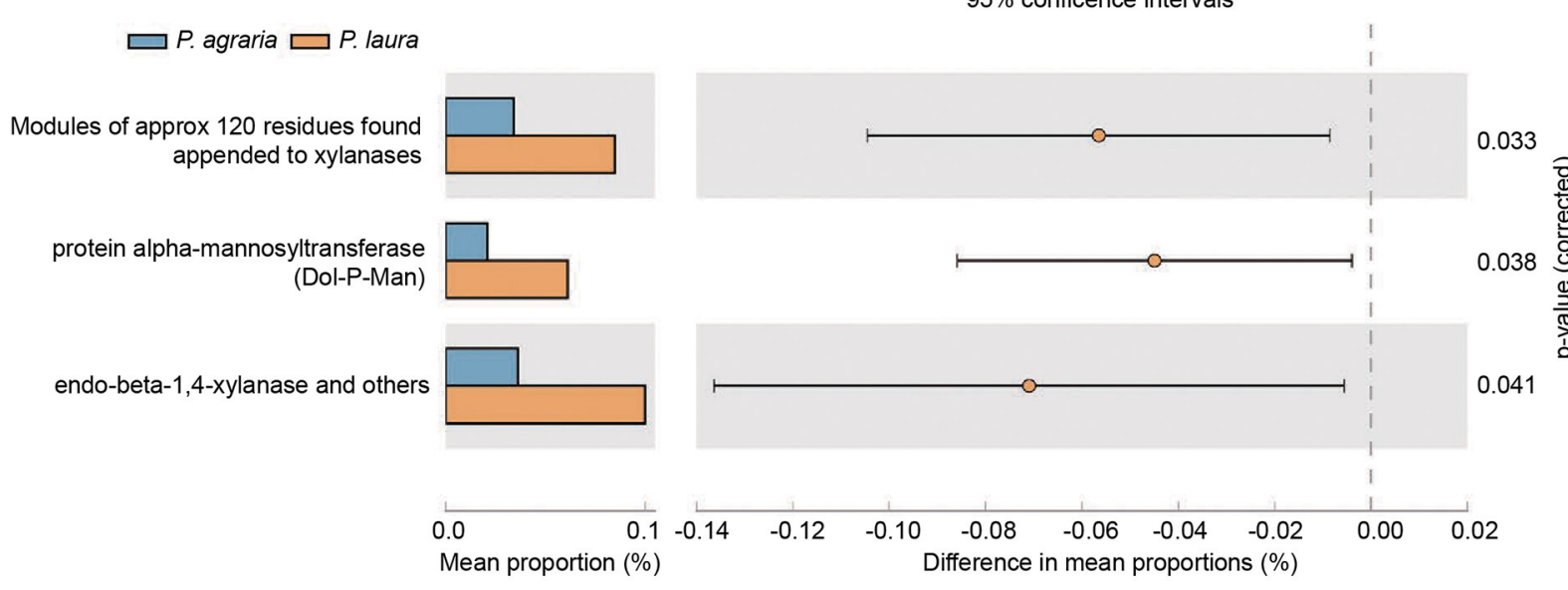

FIGURE 4 | Functional annotation of the genes in gut bacteria of the two Pardosa species. (A) Genes that mapped to the COG category were annotated using eggnog. Top 20 of the categorized functional annotation enrichment predicted in the bubble chart. The size of the circle indicates the number of genes and the color represents the significance. (B) Kyoto Encyclopedia of Genes and Genomes functional category analysis was done using GhostKOALA. Rows indicate the number of genes annotated to the corresponding B-level pathway, columns in black font are the name of the classification. (C) Comparison of antibiotic resistance-associated genes in the two Pardosa species based on the resfam database. (D) Comparison of carbohydrate metabolism-associated genes in the two Pardosa species based on the carbohydrate-active enzyme database (dbCAN2). 